\title{
Laparoscopic enhanced-view totally extraperitoneal Rives-Stoppa repair (eTEP-RS) for ventral and incisional hernias - early operative outcomes and technical remarks on a novel retromuscular approach
}

\author{
Kryspin Mitura ${ }^{1}$, Anna Rzewuska ${ }^{2}$, Marzena Skolimowska-Rzewuska³, Michał Romańczuk³ ${ }^{3}$ Krystian Kisielewski³ \\ Dorota Wyrzykowska ${ }^{1}$ \\ ${ }^{1}$ Faculty of Medical and Health Sciences, Siedlce University of Natural Sciences and Humanities, Siedlce, Poland \\ ${ }^{2}$ Faculty of Medicine, Medical University of Lublin, Lublin, Poland \\ ${ }^{3}$ Department of General Surgery, Siedlce Hospital, Siedlce, Poland
}

Videosurgery Miniinv 2020; 15 (4): 533-545 DOI: https://doi.org/10.5114/wiitm.2020.99371

\begin{abstract}
Introduction: Incisional hernias can complicate up to one in four laparotomy procedures, and successful repair remains a significant clinical challenge for surgeons. Recently, the surgical technique of ventral hernia repair (eTEP-RS) has been introduced.

Aim: To present early results in treating patients with ventral hernia using the eTEP-RS technique and to discuss key technical aspects affecting the safety and efficiency of repair.

Material and methods: A prospective study of early outcomes was conducted for all ventral hernia patients (hernia orifice between 4 and $8 \mathrm{~cm}$ ) who underwent eTEP-RS between March 2019 and July 2020.

Results: As of July 2020, we performed a total of 11 eTEP-RS procedures. The mean duration of the surgery was $204 \mathrm{~min}$ (158 to 295). The average size of the treated defect in the transverse dimension was $5.8 \mathrm{~cm}$, and the defect area was $38.5 \mathrm{~cm}^{2}$. The average size of the mesh used was $486 \mathrm{~cm}^{2}$ (280 to 590). After an average follow-up of 7 months (1-17) there was no recurrence or major complication. Based on our initial experiences we present a detailed description of the main aspects of the surgical technique itself, as well as the essential nuances, to enable evaluation of the technique and future popularization.

Conclusions: The eTEP-RS technique is a safe alternative to open ventral hernia repair and allows for the placement of a large piece of mesh in accordance with current recommendations. Excellent knowledge of the detailed anatomy of the abdominal wall is essential for safe and effective hernia repair.
\end{abstract}

Key words: surgery, outcomes, incisional hernia, totally extraperitoneal, eTEP, ventral.

\section{Introduction}

Incisional hernias can complicate up to one in four laparotomy procedures, and successful repair remains a significant clinical challenge for surgeons [1]. The optimal method of treating ventral hernias has not been established so far, and the effectiveness of the methods in use is as low as 75-80\% [2-5]. The introduction of assumptions developed by Rives and Stoppa to general surgery in the second half of the twentieth century led to the populariza- 
tion of retromuscular and preperitoneal mesh placement in the reconstruction of abdominal wall [6-8]. Despite the unquestionable advantages associated with retromuscular mesh placement, these surgical procedures have always been associated with a large abdominal incision after a past laparotomy as well as the need to perform abdominal wall reconstruction. This resulted in complications typical of mesh reconstructive surgery, such as mesh infections, eventration, and seromas, and consequently also led to recurrence [9]. Unfortunately, each subsequent recurrence leads to even more difficult future reconstructive surgery, which is additionally burdened with lower effectiveness. Hence, these complications have limited reconstructive procedures and made surgeons less interested in extensive abdominal reconstructions. The introduction of the laparoscopic technique to herniology in 1993 by Karl LeBlanc raised great hopes in surgeons regarding the possibility of minimally invasive intraperitoneal onlay mesh (IPOM) placement [10]. The limitation of the scope of intervention, along with the use of advanced synthetic materials for the production of mesh, resulted in a reduced incidence of infections in the operating field and significantly shortened the length of hospital stay [11]. Unfortunately, after some time, further complications were observed that were related to the unfavourable formation of intraperitoneal adhesions, both with the mesh itself and with the fixation devices [12]. For some products, the expected fusion between the mesh and the abdominal wall was not observed, inevitably leading to recurrences. As a result, interest in this surgical technique has decreased. It was only in 2015 that Igor Belyanski introduced the surgical technique of ventral hernia repair (eTEP; enhanced-view/extended totally extraperitoneal repair), which combined the advantages of retromuscular mesh placement (according to Rives et al. and Stoppa) $[13,14]$ with the benefits of a minimally invasive technique $[15,16]$. Belyanski et al. proposed using the laparoscopic technique to place the mesh directly behind the rectus abdominis muscle while reconstructing the linea alba and the hernia orifice. The basis for this technique was the eTEP technique introduced by Jorge Daes for inguinal hernia repairs 3 years earlier, which significantly improved the ergonomics of the surgery and made it possible to obtain an overview of the entire retromuscular space of the anterior abdominal wall [17]. Currently, interest in the enhanced-view totally extra- peritoneal Rives-Stoppa repair (eTEP-RS) technique is growing rapidly worldwide. Moreover, because the method was presented relatively recently (only 3 years earlier), it continues to gradually evolve, and technical modifications are being introduced to improve the course of the surgical procedure [18, 19].

The eTEP-RS surgery for ventral hernia requires excellent knowledge of the anatomy of the anterior abdominal wall and the ability to carefully and precisely dissect with laparoscopic instruments; furthermore, due to the fact that the procedure usually takes a long time, it is extremely important to pay attention to all the nuances and detailed technical aspects necessary to perform this reconstruction, which is not very ergonomic for the surgeon.

\section{Aim}

The aim of this study is to present our own early results in treating patients with ventral hernia using the eTEP-RS technique and to discuss key technical aspects affecting the safety and efficiency of this reconstructive surgery.

\section{Material and methods}

\section{Study design}

A prospective study of early outcomes was conducted for all ventral hernia patients who underwent eTEP-RS between March 2019 and July 2020. The average postoperative follow-up duration was 7 months (from 1 to 17 months). The presence of recurrence was considered the primary endpoint. The secondary endpoints included surgery time, duration of hospitalization, re-admission to a hospital, and the presence of intraoperative complications. In addition, key elements of the procedure were described, which underwent slight modifications and were identified as important for the course of the surgery and its ergonomics.

\section{Patients and qualifications for the study}

Eleven adult patients with midline hernias qualified for eTEP-RS surgery. The demographic and clinical data of the surgical patients are presented in Table I. The inclusion criterion was a transverse dimension of the hernia orifice between 4 and $8 \mathrm{~cm}$. All dimensions of hernia defect were measured in computed tomography scans prior to a hernia repair. Multiple defects ("Swiss cheese" hernia) were calcu- 
lated as one hernia defect in total. The exclusion criteria included lesions on the skin covering the hernia sac, presence of a concurrent stoma, previous mesh repair surgery, risk of intra-abdominal compartment syndrome associated with the presence of an extensive hernia sac, and history of laparotomies in which the scar extends from the xiphoid process of the sternum to the pubic symphysis.

\section{Procedure}

\section{Patient preparation and port placement}

All patients eligible for the surgery received anticoagulant prophylaxis before and after the procedure and a prophylactic dose of an antibiotic (cephazolin) immediately before the surgery. The patients were placed on the operating table in the supine position in slight retroflexion with their arms tucked along the body.

In the case of hernias located above the umbilicus, the lower trocar position was used (Photo $1 \mathrm{~A}$ ). After dissection of the retromuscular space on one side of the hypogastrium using a balloon dissector and insertion of the first optical trocar at the level of the umbilicus on the lateral edge of the rectus muscle, a second $10 \mathrm{~mm}$ optical trocar and two $5 \mathrm{~mm}$ working ports were then inserted suprapubically (Photo $1 \mathrm{~B}$ ). Then, the operator started bottom-up dissection from the side of the patient's legs and upwards in the cephalad direction.

For hypogastric hernias and some periumbilical hernias, trocars were inserted into the epigastrium, as shown in Photo $1 \mathrm{C}$. The monitor was placed at the level of the patient's feet. The first port was inserted retromuscularly, slightly lateral to the linea alba, in the left subcostal region using a balloon dissector. Then, after creating the retromuscular space, a $10 \mathrm{~mm}$ optical trocar was inserted into this space, followed by another $5 \mathrm{~mm}$ port slightly below the first port and medially from the linea semilunaris. This allows for further dissection of the retromuscular space in the caudal direction, distally down to the pubic symphysis, and medially to the linea alba. Additionally, one more 5 or $10 \mathrm{~mm}$ port was introduced below the second port, and dissection of the retromuscular space was continued with these two working trocars. Later during surgery, after dissecting the retromuscular space in the right side of the epigastrium, a new optical trocar was inserted contralaterally in the subcostal area (Photo $1 \mathrm{D})$, and
Table I. Patient demographic data

\begin{tabular}{|lc|}
\hline Patient demographics & Mean value \\
\hline Number of patients (\%) & $11(100)$ \\
\hline Sex (male/female) (\%) & 4/7 (36.4/63.6) \\
\hline Age, mean [years] & 30.2 (range: $26.5-35.5)$ \\
\hline Body mass index, mean $\left[\mathrm{kg} / \mathrm{m}^{2}\right]$ & $3(27.7)$ \\
\hline ASA score, $n(\%):$ & $7(63.6)$ \\
\hline I & $1(9.1)$ \\
\hline III & $0(0.0)$ \\
\hline IV & $4(36.4)$ \\
\hline \begin{tabular}{l} 
Comorbidities, $n$ (\%): \\
\hline Hypertension
\end{tabular} & $2(18.2)$ \\
\hline \begin{tabular}{l} 
Type Il diabetes \\
\hline $\begin{array}{l}\text { Chronic obstructive pulmonary } \\
\text { disease }\end{array}$
\end{tabular} & $1(9.10)$ \\
\hline $\begin{array}{l}\text { Coronary heart disease } \\
\text { Smoking }\end{array}$ & $1(9.10)$ \\
\hline Cancer history & $3(27.7)$ \\
\hline
\end{tabular}

Data are presented as the number of patients (\%) or mean (standard deviation) unless otherwise stated.

dissection began from the top and proceeded downwards in the caudal direction.

\section{Retromuscular dissection}

Perfect haemostasis should be maintained during dissection of the retromuscular space within the sheath of the rectus muscle. Muscle fibres should be separated from the posterior sheath using a hook or a high-energy device (Photos 2 A, B). The lateral dissection margin is the linea semilunaris, and in this area, the intercostal and lumbar neurovascular bundles running perpendicular to the rectus muscle should be preserved. In the medial direction, the dissection should be continued to the junction of the anterior and posterior sheaths of the rectus muscle. Caudally, below the arcuate line (below the umbilicus), the posterior sheath disappears; therefore, only the peritoneum should be separated from the rectus muscle in this area until the pubic bone is fully visible, similar to laparoscopic inguinal hernia repairs.

After dissecting the retromuscular space on one side, this space should be connected to the contra- 

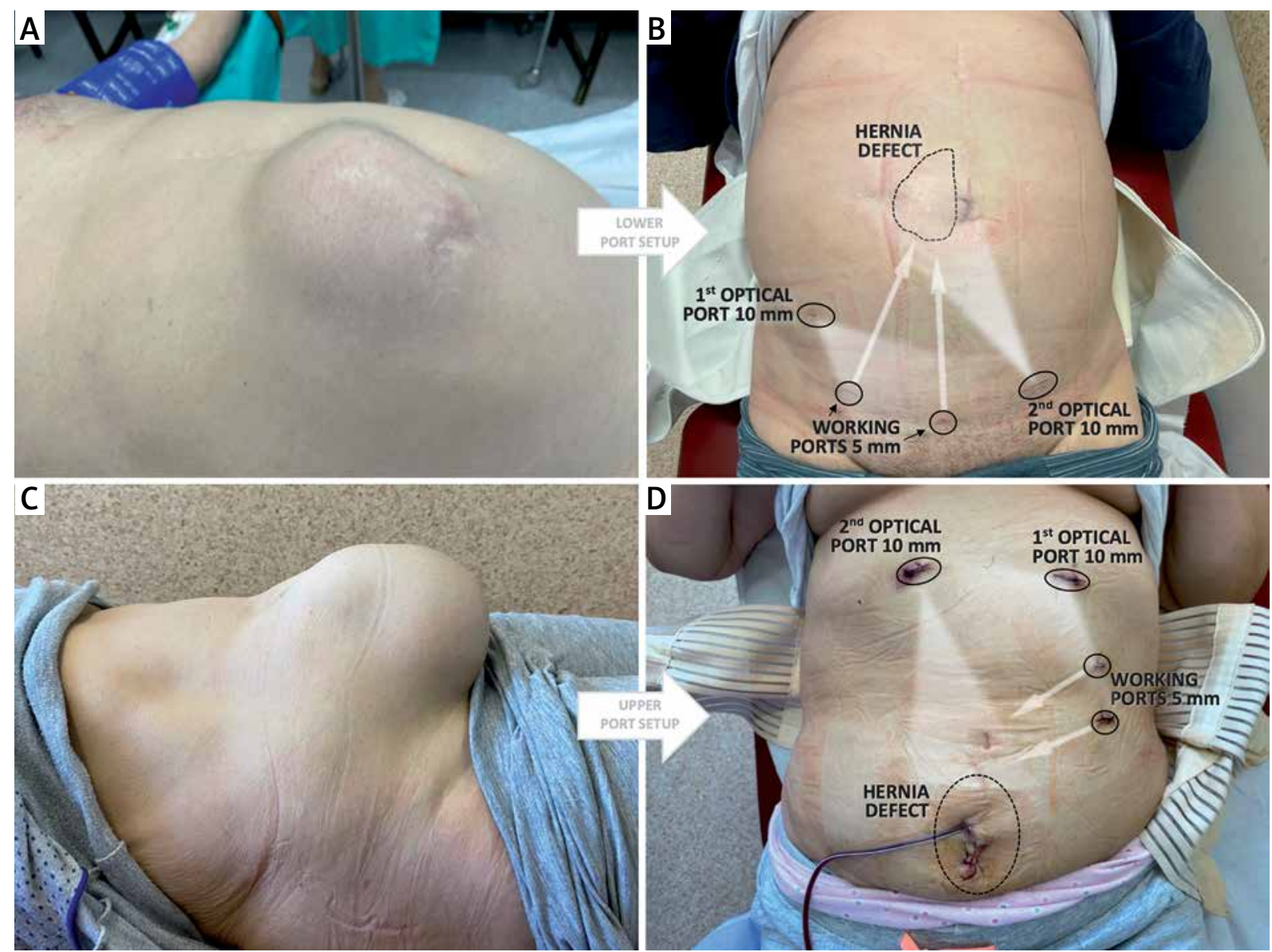

Photo 1. A - Incisional hernia in the epigastrium, B - lower port placement for epigastric hernia $\left(10^{\text {th }}\right.$ postoperative day), $\mathbf{C}$ - incisional hernia in the lower abdomen, $\mathbf{D}$ - upper port placement for hypogastric hernia ( $2^{\text {nd }}$ postoperative day)

lateral retromuscular space, but accidental opening of the peritoneum must be avoided. The posterior sheath of the rectus muscle should be incised longitudinally in the epigastrium slightly medially to the linea alba (Photos 2 C, D) and then above the falciform ligament; the posterior sheath should be exposed on the opposite side and should also be incised longitudinally, reaching the contralateral retromuscular space (Photo $3 \mathrm{~A}$ ). This space is then dissected as a whole in the same way as performed for the other side. Gradually, the dissection area was widened caudally, and the medial attachment of both posterior sheaths was cut from the linea alba (Photo 3 B), leaving the hernia sac leading to the hernia orifice (the so-called 'volcano sign') (Photo 3 C). Then, the contents of the sac were dissected from the hernia orifice (if the contents of the sac are unknown, the sac can be opened by reaching the peritoneal cavity and removing any adhesions). The hernia sac itself was not fully dissected and resected (Photo $3 \mathrm{D}$ ). After evacuation of the hernia content, all defects in the peritoneum were sutured (Photo $4 \mathrm{~A}$ ). This creates ample space that can accommodate a large piece of mesh.

\section{Linea alba reconstruction and mesh placement}

The key stage of eTEP-RS surgery is strengthening the linea alba and closing the defect in the hernia orifice. This is especially important in the case of the coexisting diastasis recti. The edges of the hernia orifice were sutured together (Photos 4 B-D), simultaneously closing them together with the medial edges of the previously cut sheaths of the rectus muscle along the entire length of the linea alba (Photo $5 \mathrm{~A}, \mathrm{~B}$ ). The use of a continuous non-absorbable barbed suture (V-loc $045 \mathrm{~cm}$, Medtronic) allows for the effective gradual closure of even wide defects. 

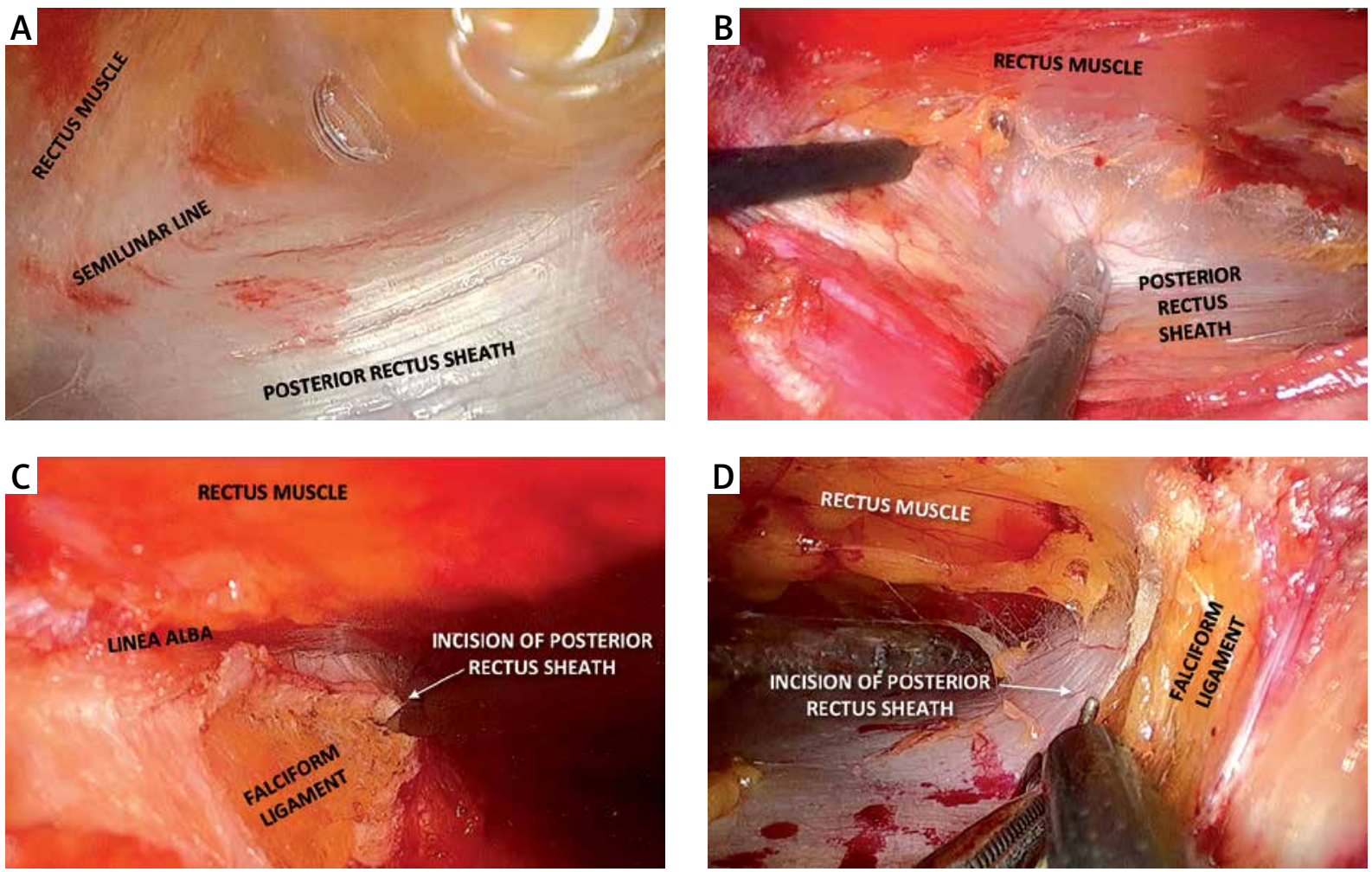

Photo 2. A - Creation of a retrorectus space with a balloon dissector, $\mathbf{B}$ - dissection of the retrorectus space with hook coagulation, $\mathbf{C}$ - medial incision of the posterior rectus sheath close to and parallel to the linea alba in the epigastrium, D - further division of the posterior rectus sheath in the caudal direction

A large piece of flat polypropylene mesh (usually $18 \times 25 \mathrm{~cm}$ ) (Photo $5 \mathrm{C}$ ) was placed in the created retromuscular space. The mesh was left with no fixation. A Redon drain was inserted through one of the trocars into the retromuscular space (Photo $5 \mathrm{D}$ ). The procedure ends with gas desufflation and suturing of the trocar wounds.

\section{Postoperative care}

Patients could drink $8 \mathrm{~h}$ after the surgery and start eating the next day. The drain was usually removed after 1-2 days. Patients were given intravenous analgesics as needed. Discharge to the outpatient clinic took place 1-3 days after surgery, depending on the extent and duration of the procedure and the patient's well-being. All patients were advised to use a wide, rigid abdominal binder for 6 weeks after surgery.

\section{Results}

As of July 2020, we performed a total of 11 eTEP-RS procedures. The treatment results are shown in Ta- ble II. The mean duration of the surgery was $204 \mathrm{~min}$ (158 to $295 \mathrm{~min}$ ). The average size of the treated defect in the transverse dimension was $5.8 \mathrm{~cm}$, and the defect area was $38.5 \mathrm{~cm}^{2}$. The average size of the mesh used was $486 \mathrm{~cm}^{2}$ (280 to 590). Although in each case we intended to use the widest possible mesh, the width of the sheaths of the rectus muscle made it necessary to trim the mesh (usually to a width of $18 \mathrm{~cm}$ ). The length of the mesh was usually $25 \mathrm{~cm}$, which corresponded to the length of the dissected space. We did not use any additional methods to fix the mesh in any of the cases. However, a mesh with self-locking hooks was used in the first 4 cases.

In 1 patient, during one of the first operations, an attempt was made to excise the whole hernia sac, which resulted in accidental skin burns with a coagulation hook and pneumoperitoneum leakage from the surgical space. The skin edges of the wound were cut out and sutured, making it possible to continue the procedure, and the wound itself healed properly. In 1 case, a complete suture of the linea alba in the epigastrium (approximately $5 \mathrm{~cm}$ 

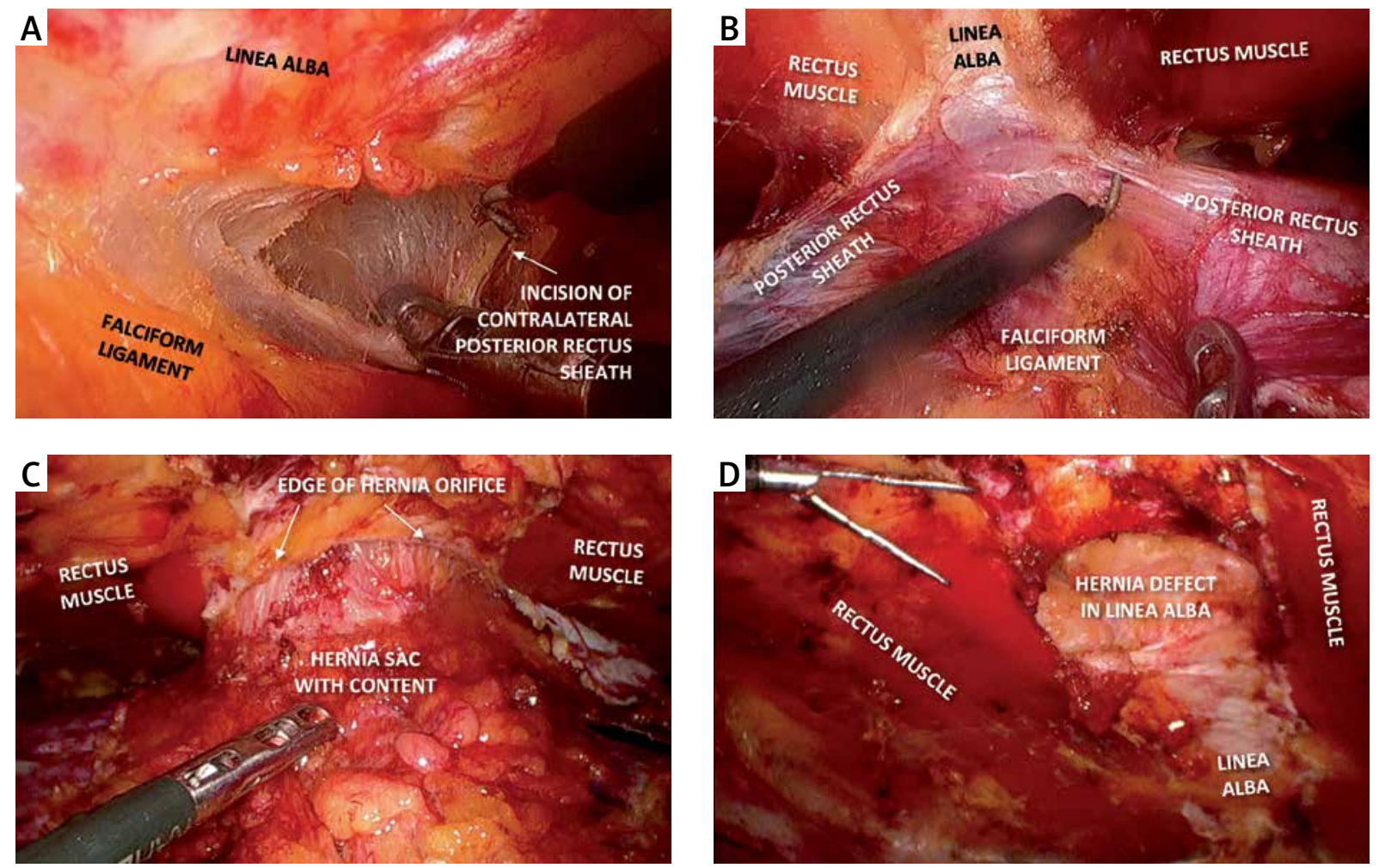

Photo 3. A - Incision of the contralateral posterior rectus sheath just above the falciform ligament (crossover), B - cranial division of the retrorectus space on both sides of the linea alba, C - "volcano sign" - hernia sac with its contents protruding through the hernia orifice, D - hernia defect in the linea alba after dissection of the retrorectus space

long) was not achieved due to improper ergonomics, but no palpable defect was found in this area in the postoperative period. One patient developed a flat haematoma of the retromuscular space on the second postoperative day, the absorption of which was monitored with ultrasound (US), and it was completely resorbed within 7 days. Two patients were diagnosed with seromas but did not require any intervention. One patient returned to the hospital 3 days after discharge because of gastrointestinal dysfunction resulting from impaired peristalsis. However, no intestinal obstruction was found, and the patient was discharged home after conservative treatment. There were no recurrences during the follow-up period. At the regular follow-up visits 1 month after the surgery, none of the patients reported any significant surgery-related pain.

\section{Technical considerations}

The absence of a detailed protocol for this procedure and the continuous evolution of this novel tech- nique necessitate a detailed description of the main aspects of the surgical technique itself, as well as the small nuances, by surgeons who have performed this procedure to enable evaluation and future popularization. We discuss the aspects of the procedure that we believe may affect the course of the surgery below. The conclusions presented below are based on our observations while mastering the method.

\section{Patient qualifications}

Due to the need for extensive separation of the posterior sheaths of the rectus muscle over its considerable length, we did not include patients with small hernias (less than $4 \mathrm{~cm}$ ) for this procedure unless they also presented with diastasis recti. It seems that for small hernias, the extent of the eTEP-RS procedure must be taken into account in the assessment of perisurgical risk. On the other hand, large ventral hernias (orifice over $8 \mathrm{~cm}$ ) are associated with a risk of the inability to suture the posterior sheaths of the recti muscles, even with partial 

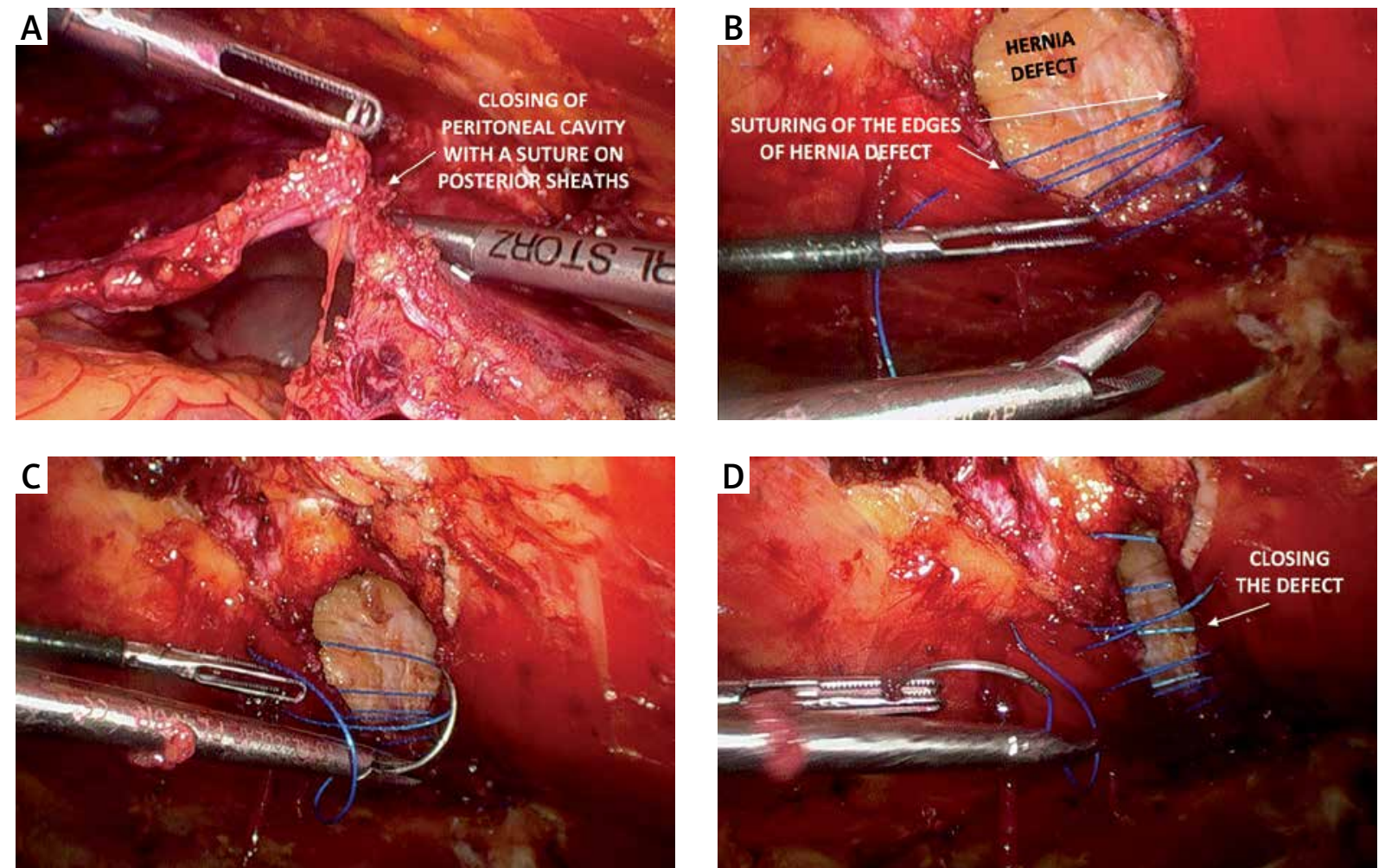

Photo 4. A - Suturing of the posterior rectus sheaths and peritoneum, B-D - closure of the hernia defect with a barbed suture with the shoe-lacing technique and gradual tightening of the suture

use of the peritoneum from the hernia sac. Suturing the posterior wall under tension increases the risk of tearing in this layer and bowel incarceration after surgery. The inability to suture this layer necessitates extension of the procedure to include another advanced procedure known as unilateral or bilateral transversus abdominis release (TAR) $[16,18]$.

\section{Patient positioning}

In the case of hernias located above the umbilicus, the operator will be standing at the patient's legs, and trocars will be inserted in the hypogastrium. Hence, the manoeuvrability of the instruments is hindered by collision with the patient's pelvis and thighs. Therefore, the table segment with the patient's legs should be lowered to the anti-Trendelenburg position so that the plane of the anterior abdominal wall in which the surgical instruments move does not interfere with the patient's thighs. When repairing hernias located below the umbilicus, the upper torso should be additionally lowered to the Trendelenburg position to avoid collision of the instruments with the chest. In addition, these manoeuvres of the operating table can increase the distance between the xiphoid process of the sternum and the pubic symphysis, which greatly facilitates dissection within the anterior abdominal wall. Preferably, the most advantageous incline of the operating table should be tested before the patient is anaesthetized to determine the limits of the table manoeuvres that are safe for the patient. If, for some reason, it is not possible to place both of the patient's arms along the torso, at least one arm should be positioned in this way (on the operator's side). A right-handed surgeon operating on a hernia in the hypogastrium should stand on the left side of the patient. At the same time, the screen (frame) separating the operating field from the anaesthetist's section with an endotracheal tube should be lowered as much as possible.

\section{Landmarks and identification}

Before the procedure, a CT examination of the abdominal cavity should be performed to assess the contents of the hernia. If the intestine is present in the hernia, additional vigilance is required when dissecting the hernia sac. Its opening should then 

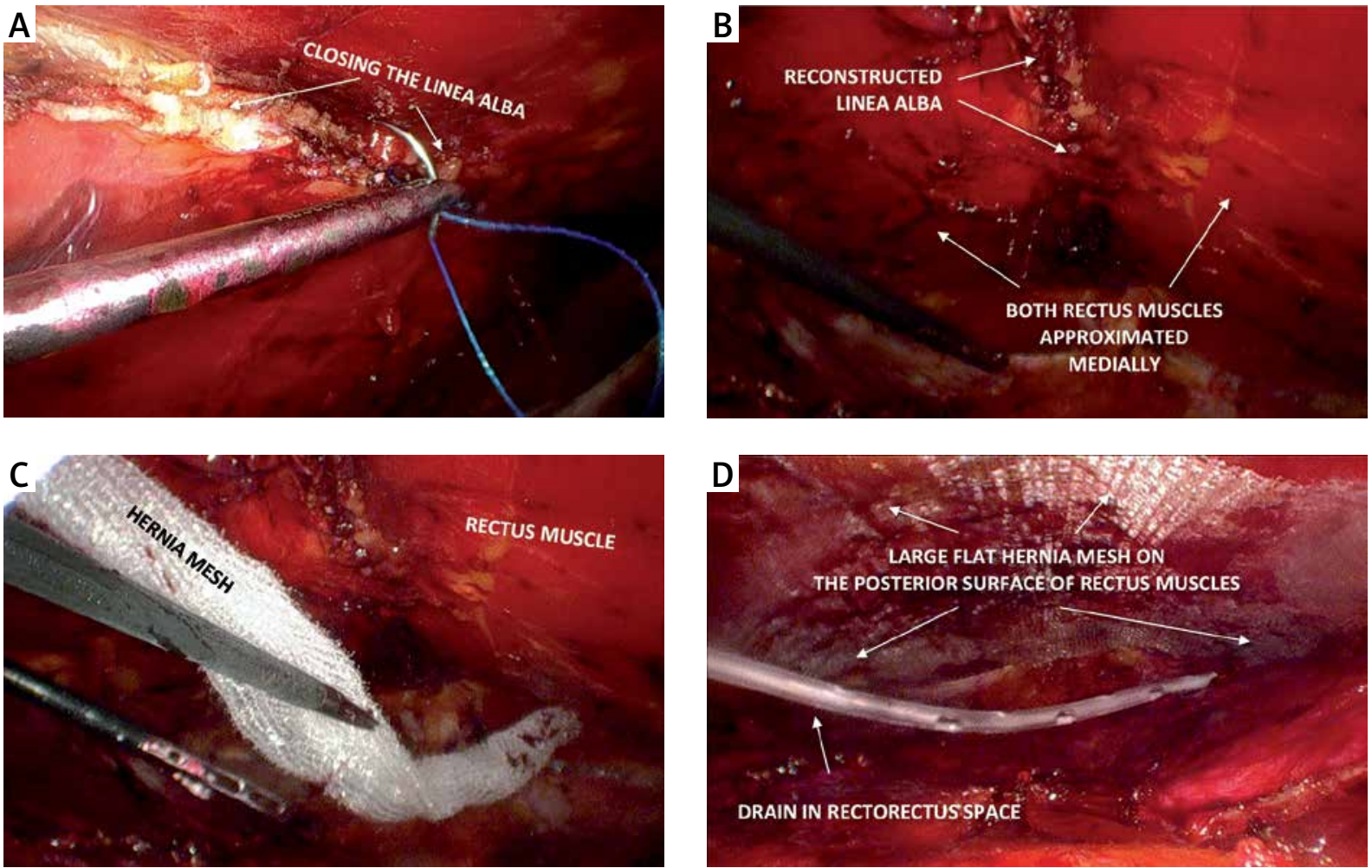

Photo 5. A, B - Closure of the linea alba with rectus muscle reapproximation, C, D - placement of a large flat piece of synthetic mesh

be considered at an early stage of the dissection of the hernia itself. Additionally, tomographic scans allow for the assessment of the size of the orifice and the width of the sheaths of the rectus muscle. If the width of the orifice is greater than half of the combined width of both sheaths, additional TAR may be required to accommodate a larger mesh size. Additionally, CT reveals accompanying defects of the abdominal wall, especially in incisional Swiss-cheese hernias. Before commencing the surgical procedure, it is advisable to mark the location of the xiphoid process of the sternum and costal margins, anterior superior iliac spines and the pubic symphysis with inguinal ligaments, the midline and both linae semilunares with a permanent marker on the skin. Portable sonographic devices can be used to determine the lateral edge of recti muscles. It is necessary to mark the location of the hernia orifice.

\section{Necessary equipment}

The basis for a safe eTEP-RS procedure in a limited space is good visibility of the surgical field. Hence, it is necessary to use oblique optics (minimum $30^{\circ}$ ) to increase the field of view by rotating the scope. A significant facilitation is the use of $5 \mathrm{~mm}$ optics, which allows for additional insight into some areas of the surgical field with $5 \mathrm{~mm}$ working ports. In addition, at the initial stage of the procedure, the dissection direction is opposite to that adopted later in surgery (in epigastric hernias, first the retropubic space is dissected in the caudal direction and then in the cephalad direction towards the hernia; in hypogastric hernias, the dissection is carried out in the caudal direction, but initially, lateral dissection is performed perpendicularly to the midline to cross over to the other side). Hence, it is much easier to use at least two monitors. The dissection of the space can be done with an ordinary monopolar hook, but the use of high-energy devices can clear a more extensive space more rapidly and reduce the amount of smoke, while reducing the risk of damaging the peritoneum with the sharp end of the hook.

\section{Port localization}

Placement of the trocars must allow for ergonomic work in various and distant areas of the ret- 
romuscular space and has to allow for convenient manipulation of the instruments during dissection of the hernia sac and suturing, which obviously are manoeuvres demanding precision. For hernias above the umbilicus, the first $10 \mathrm{~mm}$ optical port should be placed below the left costal margin, between the linea alba and the mid-clavicular line. Placing this trocar too close to the linea alba will make it impossible to see the connection of the anterior and posterior sheaths with the linea alba on that side, while positioning this port too far to the side in the sheath will hinder the ergonomics of using laparoscopic instruments inserted through this trocar later in the procedure. Another two $5 \mathrm{~mm}$ ports for working instruments should be introduced on the lateral edge of the rectus muscle slightly below the optical trocar but also above the transverse line running through the upper edge of the hernia orifice. This will allow these instruments to operate within the retromuscular space, on the opposite side and in the hypogastrium (it will be possible to dissect a space approximately $270^{\circ}$ around the hernia orifice). To insert these trocars safely and precisely, a syringe with a No. 8 injection needle is recommended. The needle is first inserted into the intended site of trocar placement, and saline is administered from the syringe. This allows for visualization of the path to pass through the rectus muscle and avoids damage to the muscles lateral to the linea semilunaris, damage to the intercostal and lumbar neurovascular bundles, and visceral damage. Notably, at this stage, the surgeon cannot elevate the muscle with another instrument. For trocars in an inferior location (in the hypogastrium), there is slightly more freedom in obtaining space and choosing the places for trocar insertion, but in this area, special attention should be paid to the inferior epigastric vessels that run along the posterior surface of the rectus muscle.

\section{Gas pressure}

After the introduction of the first optical trocar, the gas pressure should be maintained at $15 \mathrm{~mm} \mathrm{Hg}$, which allows the retromuscular space to open, facilitates dissection and inhibits oozing of blood from small vessels. However, in the event of peritoneal damage and gas entering the peritoneal cavity, the peritoneum is adversely elevated, and the retromuscular space collapses. Increasing the gas pressure at this stage does not improve visibility and should be
Table II. Perioperative data

\begin{tabular}{|c|c|}
\hline Perioperative data & Mean value \\
\hline eTEP-RS procedure $(n)$ & 11 \\
\hline Procedure time [min] & 204 (range: 158-295) \\
\hline Length of post-op stay [days] & 3.4 (range: $2-6)$ \\
\hline Hernia defect area $\left[\mathrm{cm}^{2}\right]$ : & 38.5 (range: $16.5-96.0$ ) \\
\hline Width of hernia defect $[\mathrm{cm}]$ & 5.8 (range: 4.0-8.0) \\
\hline Length of hernia defect $[\mathrm{cm}]$ & 6.3 (range: 4.0-12.0) \\
\hline Mesh area $\left[\mathrm{cm}^{2}\right]$ & 486 (range: 280-590) \\
\hline Width of mesh $[\mathrm{cm}]$ & 18.3 (range: $16.5-22.0$ ) \\
\hline Length of mesh [cm] & 25.5 (range: 20.0-30.0) \\
\hline
\end{tabular}

Hernia type, $n(\%)$ :

\begin{tabular}{|c|c|}
\hline Primary ventral hernia & $4(36.4)$ \\
\hline Incisional hernia & $7(63.6)$ \\
\hline Median & $10(90.9)$ \\
\hline Lateral & $1(9.10)$ \\
\hline Upper midline & $3(27.7)$ \\
\hline Periumbilical & $4(36.4)$ \\
\hline Lower midline & $4(36.4)$ \\
\hline Concomitant diastasis recti, $n(\%)$ & $4(36.4)$ \\
\hline \multicolumn{2}{|l|}{ Complications, $n(\%)$ : } \\
\hline Seroma & $2(18.2)$ \\
\hline Haematoma & $1(9.10)$ \\
\hline Surgical site infection & $0(0.00)$ \\
\hline Skin necrosis & $0(0.00)$ \\
\hline Wound dehiscence & $0(0.00)$ \\
\hline Prolonged ileus & $1(9.10)$ \\
\hline Bowel obstruction & $0(0.00)$ \\
\hline Respiratory complication & $0(0.00)$ \\
\hline Deep vein thrombosis & $0(0.00)$ \\
\hline Urinary infections & $0(0.00)$ \\
\hline Conversion to open repair & $0(0.00)$ \\
\hline 30-day readmission to hospital & $1(9.10)$ \\
\hline Recurrence & $0(0.00)$ \\
\hline Death & $0(0.00)$ \\
\hline Mean follow-up [months] & $7.2(1-17)$ \\
\hline
\end{tabular}

Data are presented as the number of patients (\%) or mean (standard deviation) unless otherwise stated. 
avoided. It is much more advantageous to reduce the pressure to $12 \mathrm{~mm} \mathrm{Hg}$ and apply pressure to the rising peritoneum with one of the instruments. After dissecting the retromuscular space over a wider area, spontaneous collapse of this space caused by accidental gas leakage is not as troublesome. For this reason, the hernia sac should be manipulated (stage burdened by a high risk of opening the peritoneum) only after extensively dissecting the retromuscular space and cutting the posterior sheaths of the rectus muscle. Later in the procedure, reducing the intra-abdominal pressure to approximately 9-10 $\mathrm{mm} \mathrm{Hg}$ facilitates closing of the defects in the peritoneum, and the pressure can be as low as 4-6 $\mathrm{mm} \mathrm{Hg}$ when closing the linea alba and hernia orifice, which makes it easier to bring the opposite edges of the fascia closer together. If, after suturing the hernia orifice, the working space is decreased too much, the gas pressure should be increased again. If this is not enough, the retroflexion of the operating table should be slightly reduced. Notably, because this procedure usually takes several hours, the urinary bladder becomes significantly filled, which may further reduce the available space in the working field. Hence, insertion of a urinary catheter before surgery, especially when first gaining experience or extensive procedures are performed, makes the surgery much easier.

\section{Closure of the peritoneum and fascia}

Suturing the medial edges of the dissected posterior sheaths of the rectus muscle (to close the posterior wall of the retromuscular space) is rarely possible due to the high tension in the suture line. Therefore, elements of the peritoneum should be used for this, and if possible, elements of the hernia sac should also be used. Incomplete closure of this layer, closure under tension, or leftover of small button-holes may promote penetration of the intestinal loops into the retromuscular space in the postoperative period, which may lead to early intestinal obstruction. Closing the posterior layer does not increase biomechanical resistance of the abdominal wall but only serves to create a layer of separation from the mesh. Hence, absorbable sutures can be used to close this layer. The use of barbed sutures facilitates suturing of the remaining tissues under a certain amount of tension. A key role in restoring the correct biomechanics of the abdominal wall is to restore continuity of the linea alba and close the defect in the hernia orifice. For this, it is necessary to use a thicker, non-absorbable suture, and preferably, a self-locking suture with micro-hooks. To close a wide defect, the suture is first threaded along the longer section of the fascia, and only then is the suture tightened, gradually closing the defect (shoe-lacing technique). If the medial edge of the previously cut posterior sheath cannot be visualized in the suture line, the rectus muscle should be dissected at this point so that the muscle fibres will not be included in the suture, which could lead to breaking and bleeding of their fibres.

\section{Essential blood vessels}

Particular attention should be paid to the inferior epigastric vessels, which should remain at the top of the monitor, i.e., directly on the posterior surface of the recti muscles during the dissection of the retromuscular space. To obtain a proper dissection layer, the adipose tissue accompanying these vessels should be kept in contact with the rectus muscle. A safe lateral dissection boundary should be maintained. This boundary is marked by the linea semilunaris, within which the neurovascular bundles supplying the rectus muscle are visible and are arrange:d parallel to each other. Damage to these fibres can lead to denervation of the muscle segment and its atrophy. Inadvertent dissection lateral to this line increases the risk of damage to the lateral muscles of the abdominal cavity (oblique and transverse) and may destabilize the abdominal wall. Hence, these bundles are referred to as lamp-posts. Possible release of the transverse muscle is also carried out medially from these vessels.

\section{Synthetic mesh}

Dissection of an extensive retromuscular space allows for placement of a large sheet of hernia mesh. However, it should be noted that a piece of mesh that is too large and exceeds the size of the space cannot be laid flat, which may lead to the necessity to trim it internally. As a result, the mesh may be too small and promote recurrence. The best method for precisely determining the size of the mesh is to measure the width and length of the retromuscular space with a ruler or a piece of surgical suture placed within the space. Alternatively, a less accurate indirect method may be used, i.e., measuring 
the distance between the injection needles inserted into the borders of the dissected space on the skin of the abdomen.

\section{Discussion}

Despite the enormous progress that has been made in the last two decades in the development of effective methods for the surgical treatment of inguinal hernias, the optimal treatment of ventral and incisional hernias has still not been established [6-8, 20]. The considerable variety of defects, as well as previously performed interventions, has resulted in the absence of one single effective solution. From the data available so far, it is clear that the optimal space for mesh placement is the retromuscular or preperitoneal space $[2,13,14]$. The second key aspect is the necessity to place the largest mesh possible [6]. It is now known that a strict determination of a $5 \mathrm{~cm}$ margin around the defect is insufficient for medium and large hernias. In this case, the minimum mesh width should be four times larger than the hernia orifice radius $[7,21]$. One of the dangers of ventral hernia repair is the development of infection, which may reverse the effect of the surgical procedure, require long-term treatment, and inevitably lead to recurrence $[9,21]$. It is known that the use of minimally invasive techniques helps to reduce the percentage of surgical site infections [6-8]. Taking all these data into account prompted the development of a minimally invasive technique allowing for retromuscular placement of a large sheet of synthetic mesh. This procedure, referred to as eTEP-RS, requires very precise dissection in the appropriate layer of the abdominal wall. Perfect knowledge of the anatomy of individual layers, including the muscles and fascia, is essential for navigation in the right space. The incorrect identification of an inadequate layer and its incision may lead to destabilization of the abdominal wall. In eTEP-RS procedures, there are two areas particularly susceptible to dangerous accidental damage. The first is the linea alba when crossing over to the other side over the falciform ligament. An incorrect incision in the anterior rectus sheath may separate the rectus muscle from the linea alba and cause a new hernia. Similarly, damage to the linea alba itself may create a completely new defect. The second dangerous area is the lateral edge of the rectus sheath (the linea semilunaris). As early as the earliest stage of the procedure when creat- ing the retromuscular space, the linea semilunaris may be torn if the balloon dissector is inflated too strongly. Later in the procedure, lateral dissection beyond the neurovascular bundles may also lead to detachment of the transverse or oblique abdominal muscles. Therefore, despite correct knowledge of the anatomy, it is also necessary to be aware of the functions of individual vascular and neural structures so that any iatrogenic damage does not lead to muscular atrophy or loss of muscular function, even when the intended repair is performed.

Introduction of the eTEP-RS procedure highlighted the key role of the linea alba in maintaining adequate tension of the anterior abdominal wall and supporting the muscles and mesh. The use of the laparoscopic technique allows for effective and tight suturing of the linea alba, restoring its function. This is particularly important in view of the growing knowledge of the coexistence of diastasis recti and ventral hernias, mainly primary hernias [22]. In these situations, repairing the hernia orifice only is insufficient because the defect not only affects the hernia orifice but also the entire length of the linea alba. Therefore, for a small periumbilical hernia with accompanying diastasis recti, the mesh should have appropriate margins and cover not only the umbilical hernia but also the linea alba. Additionally, the widened edges of the linea alba should be reapproximated.

Laparoscopic techniques for ventral hernia repair were first associated with the IPOM method $[10,12]$. Although this technique was technically relatively simple to perform, one of the main barriers to its popularization in the initial period was the extremely high cost of meshes and devices used for fixation. Only in subsequent assessments of the effectiveness of this treatment did surgeons realize other disadvantages, including excessive formation of peritoneal adhesions and the absence of permanent mesh adhesions to the peritoneum [23]. Contrary to IPOM, the eTEP-RS method does not require the use of additional instruments and materials. The use of a standard flat hernia mesh is quite sufficient, and fixation is not obligatory [24]. However, a factor that generates additional cost is the long duration of the repair procedure, which is typically 3 to $5 \mathrm{~h}$. These operations require long-term and meticulous dissection of the retromuscular space, which may be particularly difficult in view of the presence of adhesions after incisional hernia repairs. Similar re- 
sults were also presented by other authors. When presenting the early results of treatment in the first group of patients, Baig needed an average of 176 min to perform the surgery, and Belyansky - the originator of this method - needed an average of $141 \mathrm{~min}$ to complete the procedure $[16,25]$.

This time has shortened with progression on the learning curve. It is worth noting that despite longterm exposure, the risk of infection with this method is still minimal compared to that with open surgery because the procedure is performed in a closed space. It should be borne in mind that extensive reconstructions of the abdominal wall to treat ventral hernias require long-term procedures in open repairs as well. The use of robotic techniques in eTEP-RS resulted in a significant improvement in the ergonomics of the operation and allowed for a slight shortening of the time needed for repair. Moreover, using the DaVinci robotic platform, the average time for primary hernia surgery is approximately $2.5 \mathrm{~h}$, or $3 \mathrm{~h}$ for an incisional hernia, with the longest procedures requiring up to $5 \mathrm{~h}$ of operating the console [26].

Despite arousing great enthusiasm among surgeons and hope for effective treatment, the eTEPRS technique is introduced with great caution and respect for the outcomes. Compliance with the key principles of the surgery and identification of technical nuances that may improve the safety of the procedure are necessary for further popularization of this method and the final determination of its effectiveness. Although the postoperative discomfort and patient's complaints were not considered the endpoints of this study, we noted a more frequent possibility of early discharge following this type of surgery with perioperative management according to enhanced recovery principles [27]. The plausible reasons for these circumstances were diminished pain, greater likelihood of early mobilization and unrestricted movements. In our subjective opinion, we do not observe such possibilities after open repairs, but we did not evaluate this issue in the current study. This aspect should also be taken into account in the future in a study with a larger group of patients.

To the best of our knowledge, this is the first report evaluating the results of eTEP-RS for ventral hernia repairs in Poland. Despite the small group analysed, the preliminary results are promising. Hence, evaluations of long-term treatment outcomes in a larger group of patients will allow for more valuable conclusions. However, to collect a sufficiently large group of patients, it is necessary to perform this procedure in accordance with a similar protocol while using technical aspects described in this analysis, which may help further popularize this innovative surgical method. The small size of the study group, short follow-up period and lack of comparisons with other treatment methods used so far are disadvantages of this study. However, the dynamic development of this surgical method and its recent implementation have not allowed us to conduct research of high evidential value.

\section{Conclusions}

The eTEP-RS technique is a safe alternative to open ventral hernia repair and allows for the placement of a large piece of mesh in accordance with current recommendations. Excellent knowledge of the detailed anatomy of the abdominal wall is essential for safe and effective hernia repair. Compliance with certain rules of the eTEP-RS operation facilitates improved ergonomics for this procedure.

\section{Conflict of interest}

The authors declare no conflict of interest.

\section{References}

1. Mudge M, Hughes LE. Incisional hernia: a 10 year prospective study of incidence and attitudes. Br J Surg 1985; 72: 70-1.

2. Burger JW, Luijendijk RW, Hop WC, et al. Long-term follow-up of a randomized controlled trial of suture versus mesh repair of incisional hernia. Ann Surg 2004; 240: 578-83

3. Korenkov M, Sauerland S, Arndt M, et al. Randomized clinical trial of suture repair, polypropylene mesh or autodermal hernioplasty for Incisional hernia. Br J Surg 2002; 89: 50-6.

4. Paul A, Korenkov M, Peters S, et al. Unacceptable results of the Mayo procedure for repair of abdominal incisional hernias. Eur J Surg 1998; 164: 361-7.

5. Wheeler AA, Matz ST, Bachman SL, et al. Retrorectus polyester mesh repair for midline ventral hernias. Hernia 2009; 13 : 597-603.

6. Bittner R, Bingener-Casey J, Dietz $U$, et al. Guidelines for laparoscopic treatment of ventral and incisional abdominal wall hernias (International Endohernia Society [IEHS]) - part 1. Surg Endosc 2014; 28: 2-29.

7. Bittner R, Bingener-Casey J, Dietz $U$, et al. Guidelines for laparoscopic treatment of ventral and incisional abdominal wall hernias (International Endohernia Society [IEHS]) - part 2. Surg Endosc 2014; 28: 353-79

8. Bittner R, Bingener-Casey J, Dietz U, et al. Guidelines for laparoscopic treatment of ventral and incisional abdominal wall hernias (international Endohernia Society [IEHS]) - part III. Surg Endosc 2014; 28: 380-404. 
9. Munoz-Rodriguez JM, Lopez-Monclus J, San Miguel Mendez C, et al. Outcomes of abdominal wall reconstruction in patients with the combination of complex midline and lateral incisional hernias. Surgery 2020; 168: 532-42.

10. LeBlanc KA, Booth WV. Laparoscopic repair of incisional abdominal hernias using expanded polytetrafluoroethylene: preliminary findings. Surg Laparosc Endosc 1993; 3: 39-41.

11. Mitura K, Skolimowska-Rzewuska M, Rzewuska A, Wyrzykowska D. Is mesh always necessary in every small umbilical hernia repair? Comparison of standardized primary sutured versus patch repair: retrospective cohort study. Hernia 2020 Mar 19. doi: 10.1007/s10029-020-02170-1.

12. Mitura K, Skolimowska-Rzewuska M, Garnysz K, et al. Outcomes of bridging versus mesh augmentation in laparoscopic repair of small and medium midline ventral hernias. Surg Endosc 2017; 31: 382-8.

13. Rives J, Pire JC, Flament JB, et al. Treatment of large eventrations (apropos of 133 cases). Minerva Chir 1977; 32: 749-56.

14. Stoppa RE. The treatment of complicated groin and incisional hernias. World I Surg 1989; 13: 545-54.

15. Belyansky I, Zahiri HR, Park A. Laparoscopic transversus abdominis release, a novel minimally invasive approach to complex abdominal wall reconstruction. Surg Innov 2016; 23: 134-41.

16. Belyansky I, Reza Zahiri H, Sanford Z, et al. Early operative outcomes of endoscopic (eTEP access) robotic-assisted retromuscular abdominal wall hernia repair. Hernia 2018; 22: 837-47.

17. Daes J. The enhanced view-totally extraperitoneal technique for repair of inguinal hernia. Surg Endosc 2012; 26: 1187-9.

18. Radu VG, Lica M. The endoscopic retromuscular repair of ventral hernia: the eTEP technique and early results. Hernia 2019; 23: $945-55$

19. Ramana B, Arora E, Belyansky I. Signs and landmarks in eTEP Rives-Stoppa repair of ventral hernias. Hernia 2020 May 18. doi: 10.1007/s10029-020-02216-4.

20. Sikar H, Çetin K. The influence of mesh removal during laparoscopic repair of recurrent ventral hernias on the long-term outcome. Videosurgery Miniinv 2019; 14: 366-73.

21. Blatnik JA, Brunt LM. Controversies and techniques in the repair of abdominal wall hernias. J Gastrointest Surg 2019; 23: 837-45.

22. Fiori F, Ferrara F, Gobatti D, et al. Surgical treatment of diastasis recti: the importance of an overall view of the problem. Hernia 2020 Jun 20. doi: 10.1007/s10029-020-02252-0.

23. Mitura K. New techniques in ventral hernia surgery - an evolution of minimally-invasive hernia repairs. Pol Przegl Chir 2020; 92: 48-56.

24. Pielaciński K, Puła B, Wróblewski T, et al. Totally extraperitoneal inguinal hernia repair with or without fixation leads to similar results. Outcome of randomized prospective trial. Videosurgery Miniinv 2020; 15: 1-10.

25. Baig SJ, Priya P. Extended totally extraperitoneal repair (eTEP) for ventral hernias: short-term results from a single centre. J Minim Access Surg 2019; 15: 198-203.

26. Morrell ALG, Morrell AC, Cavazzola LT, et al. Robotic assisted ETEP ventral hernia repair: Brazilian early experience. Hernia 2020 Jun 3. doi: 10.1007/s10029-020-02233-3.
27. Zychowicz A, Pisarska M, Łaskawska A, et al. Patients' opinions on enhanced recovery after surgery perioperative care principles: a questionnaire study. Videosurgery Miniinv 2019; 14: 27-37.

Received: 2.09 .2020 , accepted: 12.09 .2020 